\title{
El aporte del sector audiovisual en las industrias culturales del Ecuador
}

\section{The contribution of the audiovisual sector in the cultural industries of Ecuador}

\author{
Abel Romeo Suing Ruiz, Universidad Técnica Particular de Loja (arsuing@utpl.edu.ec) \\ Recibido:2016-10-28 Aceptado:2017-03-01
}

\begin{abstract}
Resumen
El propósito de la investigación es determinar los cambios en la producción de las Industrias Culturales del Ecuador en los últimos cuatro años, particularmente en el sector audiovisual que ha sido objeto de políticas públicas de fomento incluidas en el Plan Nacional de Desarrollo y la Ley Orgánica de Comunicación. La metodología empleada es cuantitativa y cualitativa a través de análisis de contenidos de los informes del Ministerio de Cultura y estadísticas de Cuentas Nacionales además de entrevistas a representantes del sector audiovisual del país. Las hipótesis son: 1) Se alcanzan las metas de incremento de estrenos de obras cinematográficas de producción nacional e incremento del peso relativo de las Industrias Culturales respecto al PIB señalada en el Plan Nacional de Desarrollo y 2) Los representantes del sector valoran positivamente las políticas de creación audiovisual. Los resultados muestran que se logran las metas del Plan de Desarrollo pero aún no existe una industria consolidada porque depende de la formación de audiencias y del fomento de circuitos comerciales en un contexto de creación de nuevos canales de distribución en Internet.
\end{abstract}

Palabras claves: producción audiovisual, televisión, cine, política pública.

\begin{abstract}
The aim of this research is to determine the changes in the production of cultural industries of Ecuador in the last four years, particularly in the audiovisual sector that has been the object of public policies for the promotion included in the National Development Plan and the Organic Law of Communication. The methodology used is both quantitative and qualitative through content analysis of the reports of the Ministry of Culture and National Accounts Statistics in addition to interviews to representatives of the audiovisual sector in the country. The research bypothesis are: 1) the goals of increased releases of cinematographic works of national production and increase the relative weight of the Cultural Industries to GDP indicated in the National Development Plan are reached, and 2) the representatives of the sector positively assess the policies of audiovisual creation. The results show the goals of the Plan are achieved but there is not yet an established industry because it depends on the formation of audiences and the promotion of commercial circuits in a context of creation of new distribution channels on the Internet.
\end{abstract}

Keywords: audiovisual production, television, cinema, public policy. 


\section{INTRODUCCIÓN}

La UNESCO (2016) define a las industrias culturales y creativas como "aquellos sectores de actividad organizada que tienen como objeto principal la producción o la reproducción, la promoción, la difusión y/o la comercialización de bienes, servicios y actividades de contenido cultural, artístico o patrimonial". Los bienes y servicios culturales poseen un valor económico primario derivado de su valor cultural y tienen "potencial para la creación de riqueza y empleo a través de la generación y explotación de la propiedad intelectual" (Quartesan, Romis y Lanzafame, 2007, p. 4) y de acuerdo con el profesor Ramón Zallo (1988, p. 26) son "concebidas por un trabajo creativo, organizadas por un capital que se valoriza y destinadas finalmente a los mercados de consumo, con una función de reproducción ideológicay social".

Sobre la base de las definiciones señaladas los bienes y servicios culturales abarcan un espectro de creación en el que se cuentan la publicidad, la radio y la televisión (UNESCO, 2015, p. 11). "Usualmente incluye las siguientes industrias y consumos relacionados: publicaciones impresas y electrónicas (periódicos, libros revistas, afiches, tiras cómicas, etc.), radio, cine, video (incluyendo juegos), fotografía, música (representación de espectáculo público, grabación eimpresión), televisión, publicidad e Internet (páginas web, portales)" (Mato, 2007, p. 132), de manera concreta "una serie de creaciones simbólicas que, multiplicadas en numerosas copias en soportes materiales o inmateriales, van el encuentro de sus receptores" (Bustamante, 2008, p. 21).

El inicio de los estudios sobre industrias culturales se encuentra en la Escuela de Frankfurt, donde fueron concebidas como una "categoría de enorme utilidad analítica e interpretativa, que daba cuenta de los profundos cambios en el proceso de producción y el estatus social de la cultura" (Del Valle-Rojas et al., 2012, p. 88). Max Horkheimer y Theodor Adorno desarrollaron en 1947 las expresiones "industria cultural" y "consumo cultural". "Estos autores estaban preocupados por la formación de aquello que en su texto llaman "cultura de masas", así como por el papel que atribuían a esta en cuanto a la atrofia de la imaginación y como instrumento de dominación" (Mato, 2007, p. 134).

La Escuela de Frankfurt profundizó el concepto de "cultura de masas" al identificarlo como "industria cultural" a través del cual expresa "la idea que la cultura no surge espontáneamente de las masas, sino que es fruto de un proceso específico de producción y búsqueda de rentabilidad" (De Moragas, 2011, p. 129) es decir la industria cultural es una institución de orden simbólico de la economía de mercado "motivo lucro" es necesariamente subordinado a los "motivos" publicidad y propaganda" (Bolaño, 2013, p. 145), desde la génesis de las industrias culturales y creativas "el concepto de cultura vinculado con economía constituye una de las realidades más complejas en las ciencias sociales. A fines de los ochenta la perspectiva de la cultura como un bien sujeto de comercialización [...] se acentuó" (Avilés-Ochoa y Canizalez-Ramírez, 2015, p. 186). Luego, desde los años noventa, muchas economías nacionales mostraron interés en orientar sus perfiles industriales hacia la economía cultural, "en algunos países, esta es reconocida como una de las actividades más dinámicas en cuanto a producción y empleo" (Avilés-Ochoa y Canizalez-Ramírez, 2015, p. 187).

Las industrias culturales, de la comunicación y creativas son capas de actividades estrechamente ligadas a las que también se las denomina industrias de los contenidos. Los ejes transversales de las industrias creativas son la propiedad intelectual, el conocimiento y la apropiación social de las nuevas tecnologías de la comunicación. Germán Rey apostilla que

En América Latina son imprescindibles las políticas comunicativas abiertas a actores como las radios ciudadanas, los colectivos de creación teatral, los grupos de mujeres o las asociaciones de "hip-hoppers" que combinan economía y cultura en sus pequeños emprendimientos y que todos juntos conforman uno de los tejidos comunicativos más activos, creativos e influyentes en la vida social de la región (2011, p. 89).

Pese a la importancia creciente del desarrollo de las industrias culturales, los gobiernos particularmente los latinoamericanos reconocen que hay factores como las competencias lectoras de los jóvenes que limitan su expansión. "Las lagunas del sistema de educación son presentadas como trabas al desarrollo de ciertas industrias culturales nacionales. En esta variedad de problemas, algunos emergen, por su recurrencia, como factores dominantes" (Tremblay, 1995, p. 57).

Otro elemento que condiciona el progreso de las industrias culturales es la digitalización que en el caso de la televisión vive una transición hacia las emisiones digitales, pero lo que sería una oportunidad "ha exacerbado considerablemente la competencia. La cable-distribución lo pasa 
evidentemente mejor que la televisión. Su margen de ganancia ha sido considerable en el transcurso del último decenio" (Tremblay, 1995, p. 51).

Según la Organización para la Cooperación y el Desarrollo Económico las industrias culturales son un puntal fundamental en la producción de sus países miembros "con un crecimiento anual que oscila entre 5\% y 20\%" (Avilés-Ochoay Canizalez-Ramírez, 2015, p. 188). En 2003 las industrias culturales aportaron con el 2,6\% del PIB de la Unión Europea lo cual representó un agregado "por encima de la economía comunitaria e importante si se compara a este ramo con otros sectores tradicionales y todavía más significativos si recordamos que el 3,1 por ciento de la población de la UE trabaja en estas industrias" (Vásquez, 2009, p. 75).

Las ganancias de las industrias culturales y creativas

En todo el mundo son superiores a las de los servicios de telecomunicaciones, dentro de ellas los sectores que más ganan son la televisión, artes visuales; y, periódicos y revistas [...] Con 29,5 millones de puestos de trabajo, emplean el $1 \%$ de la población activa del mundo. Los tres principales empleadores son las artes visuales, libros y la música (Ernst \& Young, 2015, p. 8).

En América Latina la televisión es la industria cultural líder, sus ingresos son de casi un tercio del sector. "Los latinoamericanos se deleitan con los contenidos audiovisuales. En promedio, gastan más de cinco horas al día viendo la televisión [... L La penetración de la televisión de pago se encuentra entre las más altas del mundo" (Ernst \& Young, 2015, p. 63).

En Ecuador "el sector cultural requiere y provoca también intercambios y movimientos en el área económica $[. .$.$] es capaz de generar empleos$ en varios campos y etapas de la producción [sin embargo] es un país evidentemente importador de bienes y servicios culturales" (MásQMenos, 2013). En el año 2010 "las actividades culturales contribuyeron al 4,76\% PIB (y 2,2\% del total de la población ocupada trabaja en actividades culturales) [...] el 57,4\% de esta contribución proviene de las actividades culturales centrales y el 42,6\% de actividades de apoyo o equipamiento" (UNESCO, 2014, p. 6).

Las políticas públicas de comunicación y cultura ejecutadas en Ecuador en el último quinquenio destacan el potencial y los limites de las industrias culturales, de acuerdo con el Plan Nacional del Buen Vivir 2013-2017 la ausencia de "reconocimiento del inmenso potencial de la articulación entre la transformación de la matriz productiva y los procesos de creación artística y cultural ha inhibido el aprovechamiento de las industrias culturales y el desarrollo de la economía de la cultura" (Senplades, 2013). Es por ello que el Plan de Desarrollo vigente convoca a abrir espacios para aprovechar las características de estas industrias.

Ancladas al quinto objetivo del Plan Nacional del Buen Vivir2013-2017seencuentran las metas quelas políticas públicasdecomunicaciónycultura proponenalcanzar:

\section{- $\quad$ 5.4. Incrementar el número de estrenos} de obras cinematográficas independientes de producción y coproducción nacional en salas de cine comercial a 15.

- $\quad$ 5.6. Incrementar el peso relativo de las industrias culturales con respecto al PIB (solo incluye los sectores libros, publicaciones y audiovisuales) al $0,95 \%$.

En Ecuador, el papel de la cultura está vinculado a "la afirmación de una identidad nacional y al fortalecimiento de las diferentes identidades y del multiculturalismo [...] el desarrollo tiene un sentido amplio, incluyendo no solo el crecimiento económico, sino también el logro de "Sumak Kawsay", que significa "vida plena" en quechua" (UNESCO, 2014, p. 5), por lo tanto es necesario "fortalecer los valores culturales desde las industrias creativas" (Vernimmen, 2013).

Articulada a estas políticas está la Ley Orgánica de Comunicación que fue aprobada en 2013 y que en su artículo 97 señala que "los medios de comunicación audiovisual, cuya señal sea de origen nacional, destinarán de manera progresiva, al menos el 60\% de su programación diaria en el horario apto para todo público, a la difusión de contenidos de producción nacional" (Registro Oficial, 2015).

El propósito de la investigación es determinar los cambios en la producción de las Industrias Culturales del Ecuador en los últimos cuatro años, particularmente en el sector audiovisual que ha sido objeto de políticas publicas de fomento incluidas en el Plan Nacional de Desarrollo y la Ley Orgánica de Comunicación. Las hipótesis son:

1) Se alcanzan las metas de incremento de estrenos de obras cinematográficas de producción nacional e incremento del peso relativo de las Industrias Culturales respecto al PIB señalada en el Plan Nacional de Desarrollo. 2) Los representantes del sector valoran de forma positiva las políticas públicas de 
creación audiovisual pero ven factores institucionales que limitan su sostenibilidad.

de creación audiovisual pero ven factores institucionales que limitan su sostenibilidad.

\section{METODOLOGÍA}

La metodología empleada es cuantitativa y cualitativa a través del análisis de información estadística y entrevistas. Se han revisadolos informes del Ministerio de Cultura y Patrimonio del Ecuador, Consejo Nacional de Cine, Cuentas Nacionales publicadas por el Banco Central del Ecuador, Información de la Superintendencia de Compañías de Ecuador y artículos referidos a "ranking" anuales de empresas publicados en medios de comunicación masiva. El sector estudiado corresponde a la categoría J Información y comunicación de la Clasificación Internacional de la Industria Uniforme (CIIU) que emplea la Superintendencia de Compañías Ecuador, específicamente a los apartados:
J58- Actividades de publicación.

J59- Actividades de producción de películas cinematográficas, videos y programas de televisión, grabacióndesonidoyedición demúsica J60-Actividades de programación y transmisión.

Quedan fuera las actividades:

J61- Telecomunicaciones.

J62- Programación informática, consultaría de informática y actividades conexas. J63- Actividades de servicio de información.

Las entrevistas fueron realizadas a dos representantes de instituciones públicas del sector cinematográfico y audiovisual ecuatoriano, y a un realizador independiente (cuadro 1).

Las entrevista versan sobre los factores que influyen en el consumo audiovisual y los retos de la industria audiovisual nacional.

Cuadro 1. Perfiles de entrevistados

\begin{tabular}{|c|c|c|}
\hline Nombre & Actividades & Descripción \\
\hline Wilma Granda & $\begin{array}{c}\text { Directora de la Cinemateca Nacional } \\
\text { de la Casa de la Cultura Ecuatoriana. }\end{array}$ & $\begin{array}{c}\text { La Cinemateca Nacional del Ecuador fue fundada el de diciembre } \\
\text { de 1981, se dedica a la preservación y difusión de las obras y } \\
\text { registros audiovisuales ecuatorianos. }\end{array}$ \\
\hline Isabel Mena & $\begin{array}{c}\text { Directora Ejecutiva del Consejo } \\
\text { Nacional de Cine }\end{array}$ & $\begin{array}{c}\text { El Consejo Nacional de Cine es la institución creada para ejecutar } \\
\text { las políticas encaminadas a fortalecer la cultura cinematográfica } \\
\text { del país. }\end{array}$ \\
\hline Daniel Granda & Productor y realizador audiovisual & Trabaja en realizaciones independientes. \\
\hline
\end{tabular}

Elaboración: Propia

\section{RESULTADOS}

De acuerdo con las estadísticas del Ministerio de Cultura y Patrimonio de Ecuador, y del
Consejo Nacional de Cine, los estrenos de obras cinematográficas por año desde 2012 se han incrementado en una tasa media de $111 \%$ (Figura 1).

Figura 1. Estrenos de obras cinematográficas independientes de producción y coproducción nacional

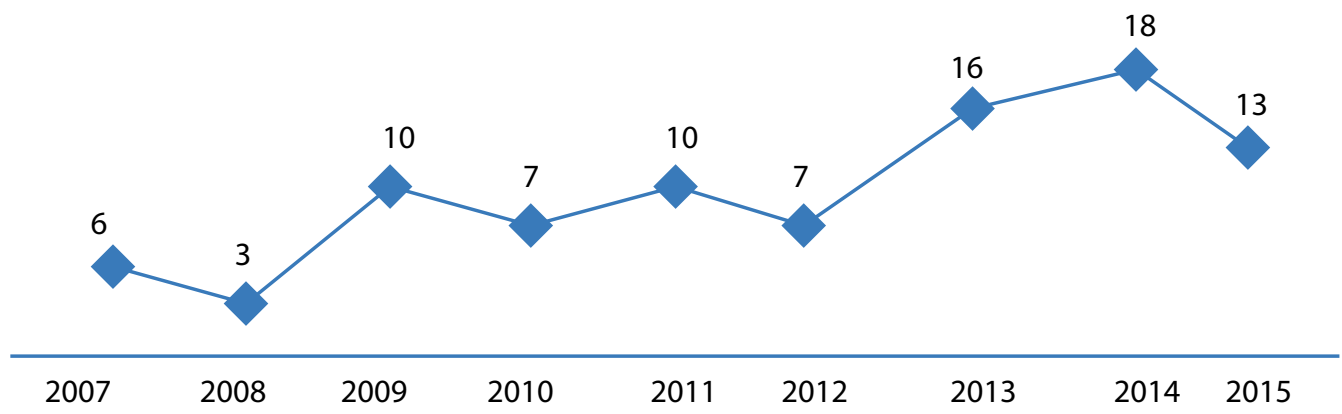

Fuente: Ministerio de Cultura y Patrimonio de Ecuador y Consejo Nacional de Cine

Elaboración: Ministerio de Cultura y Patrimonio. Coordinación General de Planificación - Proyecto Sistema Nacional de Cultura 
La relación porcentual de personal y utilidades de las industrial culturales respecto al total de industrias de Ecuador ha disminuido entre 2013 y 2015, en este periodo no han existido utilidades aunque parecería que hay bonanza sobre la base de las cifras de crecimiento de los ingresos (figura 2). Estas disminuciones de ganancias de las industrias culturales de Ecuador ocurren sobre todo en las empresas de televisión y prensa. Cuando se revisa la evolución de ingresos y personal ocupado en las mayores estaciones de televisión (Ecuavisa, GamaTV, RTS, Canal 10) y prensa (El Comercio, El Universo,

El Telégrafo y los medios impresos de Granasa) se observan descensos y pérdidas (figuras 3 y 4 ) lo que en cambio no ocurre con las empresas de publicidad.

Figura 2. Relación porcentual de industrias culturales

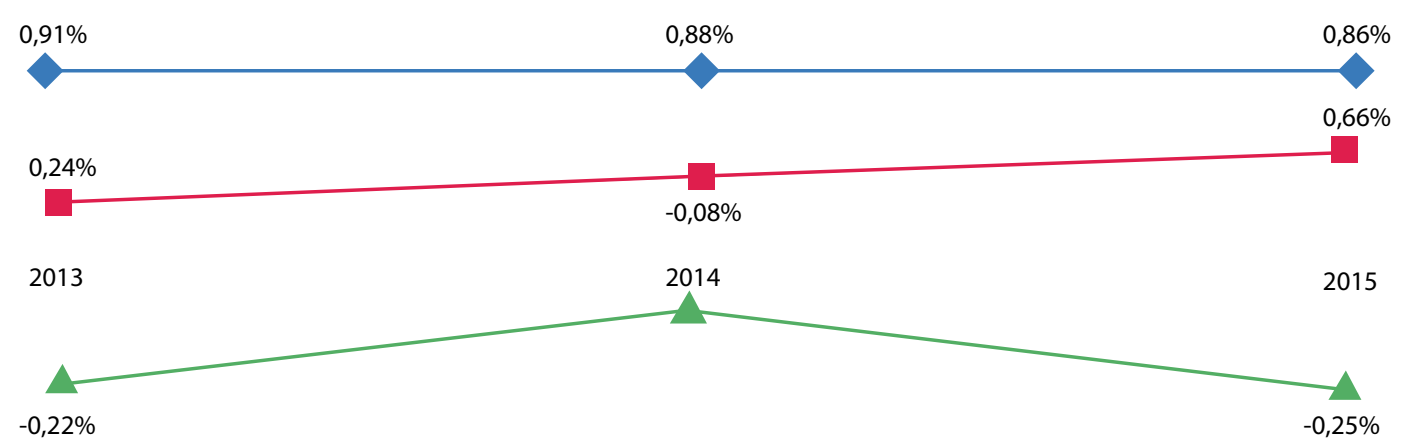

Fuente: Superintendencia de Compañías de Ecuador Elaboración: Propia

Figura 3. Ganancias de empresas grandes (cifras en millones de \$)

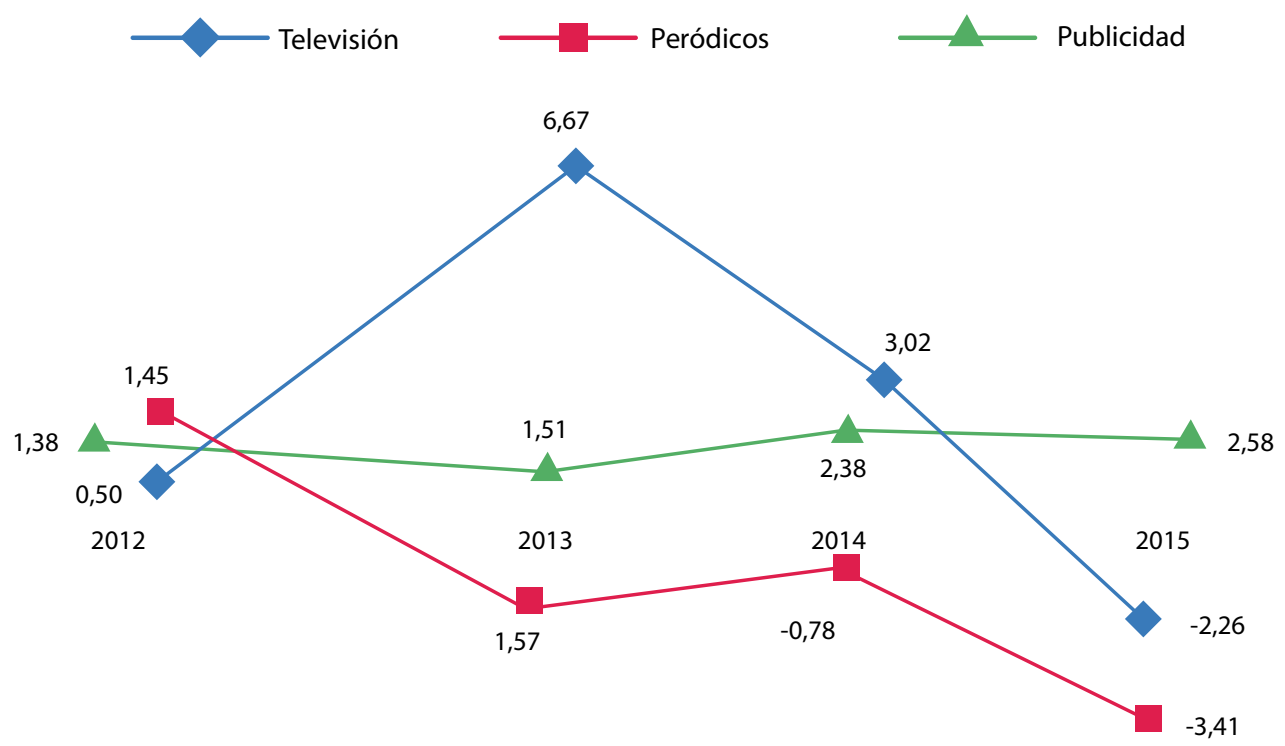

Fuente: Ranking de empresas 2016

Elaboración: Propia 
Figura 4. Personal de empresas grandes

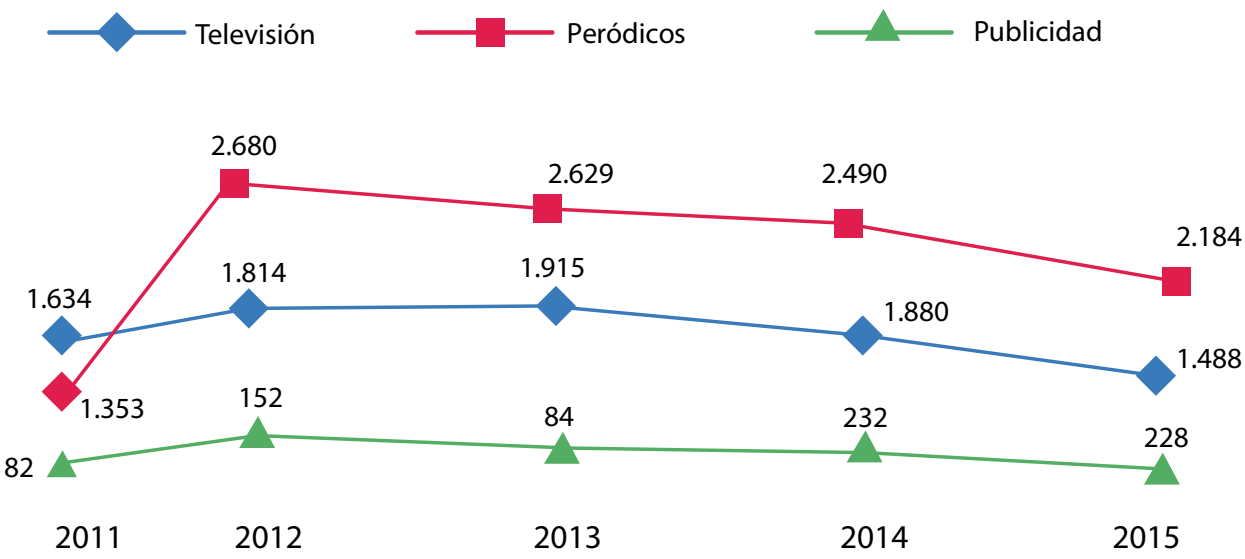

Fuente: Ranking de empresas 2016

Elaboración: Propia

Pese a las disminuciones de ingresos y personal ocupado las industrias culturales de Ecuador presentan una tasa promedio de $3.1 \%$ respecto al
PIB de Ecuador en los últimos nueve años (figura 5), período en el que han crecido en más de un punto porcentual en la composición del PIB.

Figura 5. Estructura porcentual de Industrias Culturales respecto al PIB (a precio de 2007)

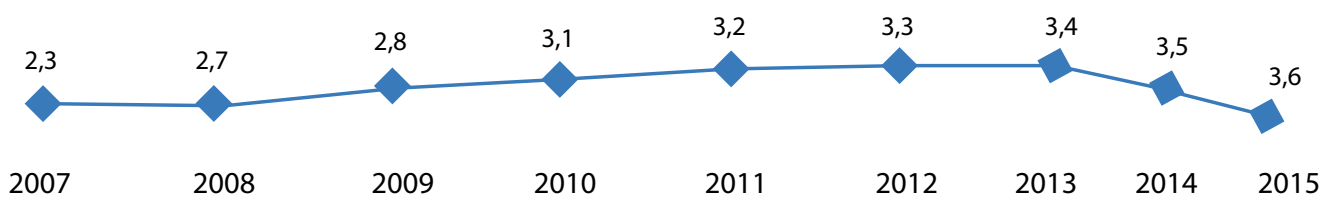

Fuente: Banco Central del Ecuador

Elaboración: Propia

La valoración cualitativa de las industrias cultuales se presenta a continuación. Wilma Granda señala que en el contexto actual uno de los factores que más influye en el consumo audiovisual es la demografía que exige diversificar y formar al público a través de la democratización de los espacios en las salas de cine del país para que las audiencias, movimientos sociales o colectivos de realizadores y distribuidores independientes hagan uso de los ellos. En el mismo sentido Isabel Mena manifiesta que hay elementos que influven en la cultura audiovisual como la falta de películas ecuatorianas en las salas de cine comercial, las razones de esta ausencia estaría en los tamaños de las salas, horarios, precios de entradas y distribuciones a través de televisión e Internet. Daniel Granda añade que plataformas como Netflix o Vimeo condicionan los consumos audiovisuales de los jóvenes.

De acuerdo con Wilma Granda no hay una industria audiovisual en Ecuador, todavía está en construcción, en todos los procesos, del guion de la preproducción, es necesario apuntar a experiencias de formación que permitan valorar nuestras historias, escribirlas y construirlas en el audiovisual. Para Isabel Mena se debe diversificar la oferta para llegar a diferentes públicos, en la variedad está la clave. Daniel Granda precisa que estamos en un nivel artesanal de cine porque trabajamos con presupuestos muy reducidos, el cine nacional ha sido bastante criticado por no llegar a los públicos, parece que se ha producido de acuerdo con el gusto de los directores en lugar del gusto del público.

Wilma Granda señala que para que el público vaya al cine primero debe romperse el monopolio norteamericano, aunque Isabel Mena presenta otras herramientas como Internet yenfatiza en la formación de la audiencia que debe ocurrir desde la infancia; en este aspecto coincide Daniel Granda al señalar que la competitividad de Hollywood en producción a gran escala y con altos presupuestos de publicidad incide en la formación del público. Wilma Granda manifiesta que para contar con una industria audiovisual nacional se debe reconstruir 
la memoria estética y la autoestima, el público debe crear hábitos culturales como leer, escuchar música, asistir a exposiciones, es decir es un trabajo colectivo con otros sectores culturales. Para Isabel Mena es necesario que las políticas públicas consideren que el cine, como industria, no debe depender únicamente de la asignación del Presupuesto General del Estado. En palabras de Daniel Granda vivimos una transición, en los últimos años se estrenan películas anualmente pero se necesita aprender a contar historias, hacer que el relato sea un elemento importante en el cine ecuatoriano.

\section{CONCLUSIONES}

En economías cada vez más diversificadas hacia los sectores de servicios y de gestión del conocimiento se esperaría que existan industrias culturales fortalecidas mucho más cuando existe un marco de políticas publicas que promueven el patrimonio cultural e intangible; sin embargo, parece que no hay crecimientos armónicos de estas en Ecuador a la luz de las estadísticas entregadas por la Superintendencia de Compañías.

Las industrias culturales de Ecuador particularmente de televisión y prensa no muestran condiciones para un desarrollo sostenido e incluso su permanencia estaría afectada en razón de los descensos de puestos de trabajo y utilidades. La disposición de fomento a la producción nacional que incluye la Ley Orgánica de Comunicación no se refleja en crecimiento de los ingresos de la industria televisiva nacional.

Además, hay un crecimiento importante del número de estrenos de obras cinematográficas de producción y coproducción nacional como consecuencia de las políticas culturales de los entes públicos, entre otros factores. El aporte de las industrias culturales al PIB de Ecuador en los últimos 10 años es $3,1 \%$ en promedio y muestra crecimiento constante, excepto el último año que desciende $0,1 \%$, es un crecimiento valioso pero aún lejano de las tasas de 2,6\% de la Comunidad Europea.

Un elemento coyuntural que afecta a las industrias culturales y en general a la economía de Ecuador es la reducción de los ingresos petroleros a la caja fiscal ocurridos desde 2014, en promedio las industrias ecuatorianavieron disminuidos susingresos en $10 \%$, las medidas de política económica que tomó el gobierno como los cupos a las importaciones y salvaguardias han provocado contracción en la economía.
La lectura de los expertos es que las industrias culturales de Ecuador aún no están consolidadas y dependen de la formación de audiencias y del fomento de los circuitos comerciales en un contexto en que deben aproximar las creaciones a los nuevos canales de distribución en Internet. El indicador que permitiría hablar de una industria cultural madura es la menor dependencia de los fondos públicos.

Las metas propuestas en el quinto objetivo del Plan Nacional del Buen Vivir 2013-2017 se alcanzan, el número de estrenos de obras cinematográficas independientes de producción y coproducción nacional en salas de cine comercial superan las 15 por año (figura 1) y existen incrementos en el peso relativo de las industrias culturales con respecto al PIB (sólo incluye los sectores libros, publicaciones y audiovisuales) como se presenta en el figura 5, por lo tanto la primera hipótesis de la investigación seacepta.

La segunda hipótesis también es aceptada porque de acuerdo a los representantes del sector las políticas públicas de promoción de creación audiovisual son positivas pero falta construir condiciones institucionales que hagan sostenible al audiovisual, además y de acuerdo a los diagnósticos del Plan Nacional de Desarrollo las industrias cultuales a través del audiovisual puede contribuir a la meta de buen vivir señalada como "Sumak Kawsay".

\section{REFERENCIAS BIBLIOGRÁFICAS}

Avilés-Ochoa, E. y Canizalez-Ramírez, P. (2015). Industrias culturales y crecimiento económico. Un modelo para el estudio del surgimiento de clusters creativos. Economía, Sociedad y Territorio, 15(47), 185-216.

Banco Central del Ecuador (2016): Información Económica. Recuperado de: https://www.bce. fin.ec/index.php/component/k2/item/788

Bolaño, C. (2013). Industria cultural, información y capitalismo. Barcelona: Gedisa Editorial

Bustamante, E. (2008). Hacia un nuevo sistema mundialdecomunicación.Barcelona:GedisaEditorial

Consejo Nacional de Cine (2016) Cine en cifras. Recuperado de: http://www. cncine.gob.ec/cncine.php?c=1616

De Moragas, M. (2011). Interpretar la comunicación. Barcelona: Gedisa Editorial 
Del Valle-Rojas, C., Nitrihual-Valdebenito, L. y Mayorga-Rojel, A. J. Mayorga-rojel, A. J. (2012): Elementos de economía política de la comunicación y la cultura: hacia una definición y operacionalización del mercado de los medios. Palabra Clave, 15 (1),82-106. Recuperado dehttp:// www.redalyc.org/articulo.oa?id $=64923563005$

MásQMenos (18 de febrero de 2013): Las industrias culturales en Ecuador. El Telégrafo. Recuperado de http://www.eltelegrafo.com.ec/noticias/ masqmenos-2/1/las-industrias-culturales-en-ecuador

Mato, D. (2007): Todas las industrias son culturales: crítica de la idea de "industrias culturales" y nuevas posibilidades de investigación. Comunicación y Sociedad, (8), 131-153. Recuperado de http://dialnet.unirioja.es/servlet/

Ministerio de Cultura y Patrimonio de Ecuador (2016): Indicadores culturales. Recuperado de: http://picultural.culturaypatrimonio.gob.ec/

Quartesan, Alessandra; Romis, Mónica y Lanzafame, Francesco (2007). Las industrias culturales en América Latina y el Caribe: desafíos y oportunidades. Departamento de Capacidad Institucional y Finanzas del Banco Interamericano de Desarrollo. Washington: BID.

Ranking de empresas 2016 (23 de septiembre de 2016). 500 mayores empresas del Ecuador. El impacto de la caída. Revista Vistazo Na 1178, pp 155-216

Registro Oficial (2015): Ley Orgánica de Comunicación. Registro Oficial Suplemento 22 de 25-jun-2013. Última modificación: 18-dic-2015

Rey, G. (2008): La otra cara de la libertad. La responsabilidad social empresarial de los medios de comunicación en América Latina. Bogotá: Fundación Nuevo Periodismo Iberoamericano.

Senplades (2013): Plan Nacional del Buen Vivir 2013 -2017. Recuperado dehttp://www.buenvivir.gob.ec/
Superintendencia de Compañías de Ecuador (2016): Portal de Información. Recuperado http://appscvs.supercias.gob.ec/ portalinformacion/sector_societario.zul

Tremblay, G.(1995): La investigación sobre las industrias culturales en una economía abierta. Comunicación y Sociedad. Universidad de Guadalajara. No (22-23), 33-69.

UNESCO (2014): Indicadores delaUNESCO de cultura para el desarrollo. Resumen analítico de Ecuador. Diversidad de la expresiones culturales. Recuperado de: http://es.unesco.org/creativity/sites/creativity/ files/cdis/resumen_analitico_ecuador_0_1.pdf

UNESCO (2015): Cultural times. The first global map of cultural and creative industries.

UNESCO - CISAC. Recuperado de http://www. UNESCO.org/new/fileadmin/MULTIMEDIA/HQ/ ERI/pdf/EY-Cultural-Times2015_Low-res.pdf

UNESCO (2016): Industrias Creativas. Oficina Regional de Educación de la UNESCO para América Latina y el Caribe. Santiago. Recuperado de http://www.UNESCO.org/ new/es/santiago/culture/creative-industries/

Vásquez, D. (2009): El debate de las industrias culturales en América Latina y la Unión Europea. The Debate About Cultural Studies in Latin American and the European Union, 15(30), 61-84. Recuperado de http://search. ebscohost.com/login.aspx?direct $=$ true $\& \mathrm{db}=$ fua\&AN $=47181227 \&$ lang $=$ es\&site $=$ ehost-live

Vernimmen, L. (2013): Las industrias culturales para el desarrollo de Ecuador. Resistencia. Recuperado de http://repositorio.uasb.edu.ec/ bitstream/10644/4353/1/10-OT-Vernimmen.pdf

Zallo, R. (1988). Economía de la comunicación y de la cultura. Madrid: Akal. 